International Journal of Medical Anesthesiology 2019; 2(1): 01-02

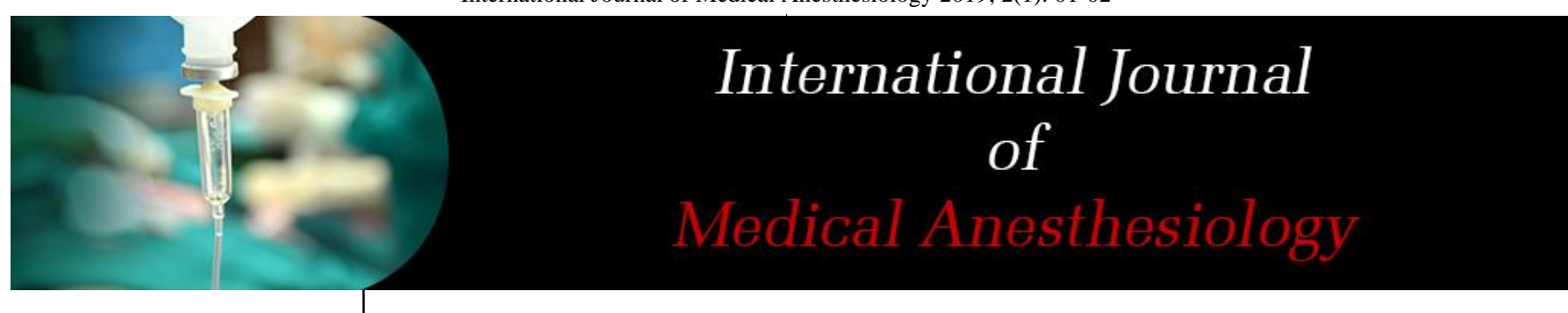

E-ISSN: 2664-3774

P-ISSN: 2664-3766

www.anesthesiologypaper.com

IJMA 2019; 2(1): 01-02

Received: 01-11-2018

Accepted: 04-12-2018

Brig Khalid Zaeem

Classified Anesthesiologist,

Military Hospital, Rawalpindi,

Pakistan

\section{Sanum Kashif}

Classified Anesthesiologist,

Military Hospital, Rawalpindi,

Pakistan

\section{Azmat Abro}

Classified Anesthesiologist, Military Hospital, Rawalpindi, Pakistan

\section{Anaesthesia for herniotomy in a pediatric patient with Criss cross heart disease}

\author{
Brig Khalid Zaeem, Sanum Kashif and Azmat Abro
}

DOI: $\underline{\text { https://doi.org/10.33545/26643766.2019.v2.i1a.16 }}$

\begin{abstract}
Background: Congenital heart disease (CHD), still placed first among common birth defects, occurs approximately 1 in 125 live births. Crisscross heart disease (CCHD) is an uncommon cardiac malformation characterized by crossing of the inflow blood streams of the two ventricles due to an apparent longitudinal twisting of the heart.

Case Report: We report a case of a 4-month-old infant, a diagnosed case of complex congenital heart disease (CCHD, VSD, pulmonary atresia and mild AR) and was on anti-heart failure treatment, admitted to our hospital for left inguinal herniotomy.

Conclusion: The challenge for anaesthetists dealing with CHD patient, coming for other than cardiac surgery depends on the patient's age, complexity of the heart lesion, coupled with patients' capacity to compensate, multiple comorbids and surgical urgency.
\end{abstract}

Keywords: Anesthesia management, Criss cross heart disease (CCHD), extra cardiac surgery

Introduction

CCHD is an extremely rare congenital cardiac anomaly characterized by abnormally rotated ventricular mass along its major axis ${ }^{[2]}$. Although it is classified as a defect, the Criss-cross heart $(\mathrm{CCH})$ is more of a spatial anomaly than a functional one, and it is possible for the heart to have relatively normal functioning. Patients usually presents with cyanotic appearance and systolic murmur on auscultation.

Case report: This is the case of 4 month old male infant, weighing $3.8 \mathrm{~kg}$ and a diagnosed case of CHD, came with left inguinal hernia. On examination, he was vitally stable, had club feet, hepatomegaly and on auscultation, systolic murmur was present at left upper sternal border, therefore, provisionally diagnosed as a case of VSD, rest of the examination was unremarkable. Treatment, he received includes Intravenous antibiotics, Tab. Spiromide and Capoten. Echo findings showed Situs Solitus, levocardia, Criss cross heart, AV/VA concordance, large inlet VSD Lt to Rt shunt and small ASD secundum. He was diagnosed as case of $\mathrm{CCH}$ with large VSD+pulmonary atresia (pseudo truncus type) and mild AR.

In the operation theatre, inhalation induction with Oxygen and Sevoflurane was used and LMA of 1.5 size inserted. Intraoperative analgesia was achieved by intravenous Ketamine $8 \mathrm{mg}$ and a caudal block with Ropivacaine. Maintenance was achieved with oxygen and Isoflurane $1 \%$. Patient remained hemodynamically stable and successfully recovered by the end of surgery. Post-operatively he was kept in ICU for hemodynamic monitoring and discharged to home on next day of surgery.

\section{Discussion}

The CCHD is an extremely rare malformation but well described morphologically. The diagnosis of CCHD is based on the axes intersection of entries of ventricles. In a normal heart these axes are virtually parallel. In CCHD, the base of the heart remains in spatial position but the ventricles appear to have been twisted along their longitudinal axis. This promotes a change in hemodynamics characterized by crossing flows through the atrioventricular valves, resulting in impression that direction of each atrium is towards contralateral ventricle ${ }^{[3]}$.

There are cases of CCHD described in the literature with discordant atrioventricular connections associated with transposition of the great vessels which results in a corrected physiological circulation, since the presence of "double mismatch", venous blood direction towards lungs and direction of arterial blood towards systemic circulation.
Corresponding Author: Sanum Kashif

Classified Anesthesiologist, Military Hospital, Rawalpindi, Pakistan 
Patients with this type of anomaly, which represents $0.05 \%$ of $\mathrm{CHD}$, may be symptomatic not because of the $\mathrm{CCH}$, but due to other related anomalies such as Ventricular septal defect, pulmonary valve obstruction and abnormalities of tricuspid valve.

The main challenge for Anaesthesiologist in CHD patient's handling, coming for other than cardiac surgery depends on patients' age, complexity of the heart lesion, coupled with patients' capacity to compensate, multiple comorbids and surgical urgency ${ }^{[4,5]}$.

\section{Conclusion}

As there are many complex factors involved, anaesthesia for extra-cardiac surgery of CHD patients, is based on confidence and experience in handling the case. Preplanning, team work and good knowledge in physiological and pharmacological principles help in successful completion of the procedure ${ }^{[6,7]}$.

\section{References}

1. Fang $\mathrm{F}$, Li ZA, Yang $\mathrm{Y}$, Zheng $\mathrm{CH}$, Lam YY. Deciphering the mysteries of crisscross heart by transthoracic echocardiography. Echocardiography. 2011; 28(1):104-8.

2. Nielsen JC, Parness IA. Anatomy of a criss-cross heart. Circulation. 2002; 106(9):e41

3. Chowdhury D. Pathophysiology of congenital heart diseases. Ann Card Anaesth. 2007; 10:19-26.

4. Frankville D. Anesthesia for children and adults with congenital heart disease. In: Lake CL, Booker PD, editors. Pediatric Cardiac Anesthesia. Philadelphia: Lippincott Williams and Wilkins, 2005, 601-32.

5. Diaz LK, Andropoulos DB. New developments in pediatric cardiac anesthesia. Anesthesiology Clin North America. 2005; 23:655-76. [PubMed]

6. White MC. Approach to managing children with heart disease for noncardiac surgery. Paediatr Anaesth. 2011; 21:522-9.

7. Walker A, Stokes M, Moriarty A. Anesthesia for major general surgery in neonates with complex cardiac defects. Paediatr Anaesth. 2009; 19:119-25. 